\title{
Fission chambers designer based on Monte Carlo techniques working in current mode and operated in saturation regime.
}

\author{
Alfonso Antolínez, David Rapisarda
}

\begin{abstract}
Fission chambers have become one of the main devices for the measurement of neutron fluxes in nuclear facilities; including fission reactors, future fusion ones, spallation sources, etc. The main goal of a fission chamber is to estimate the neutron flux inside the facility, as well as instantaneous changes in the irradiation conditions. A Monte Carlo Fission Chamber Designer (MCFCD) has been developed in order to assist engineers in the complete design cycle of the fission chambers. So far MCFCD focuses on the most important neutron reactions taking place in a thermal nuclear reactor. A theoretical model describing the most important outcomes in fission chambers design has been developed, including the expected electrical signals (current intensity and drop in potential) and, current polarization voltage character istics (sensitivity and saturation plateau); the saturation plateau is the zone of the saturation curve where the output current is proportional to fission rate; fission chambers work in this region. Data provided by MCFCD are in good agreement with measurements available.
\end{abstract}

1. Introduction

2. Monte Carlo fission chamber designer

3. Numerical model

3.1. Neutron transport

3.2. Fission fragments

3.3. Pairs production.

3.4. Electrical signal

4. Results of numerical simulations

5. Conclusions

Acknowledgments

References

\section{Introduction}

The advent of the new nuclear fusion facilities based on mag netic confinement, like ITER [1], has shown the absence of suitable instruments for on line, in core measurement of neutron fluxes in these facilities which are characterized for having high and fast neutron fluxes [2]. The signal provided by the fission chamber is used as a basis for monitoring and calculating some of the most critical parameters, such as power, in core energy distribution, fuel burn up and damage of structural materials, among others.

Cylindrical fission chambers consist of a pair of electrodes, one of which usually the anode, is coated with a fissile material, usually U235 but other fissile isotopes, such as U238, Pu242 can be used according to the neutron spectra is intended to analyze. The space between the electrodes is filled with inert gas, often argon, sometimes with a small percentage (4\%) of nitrogen or other gases. 


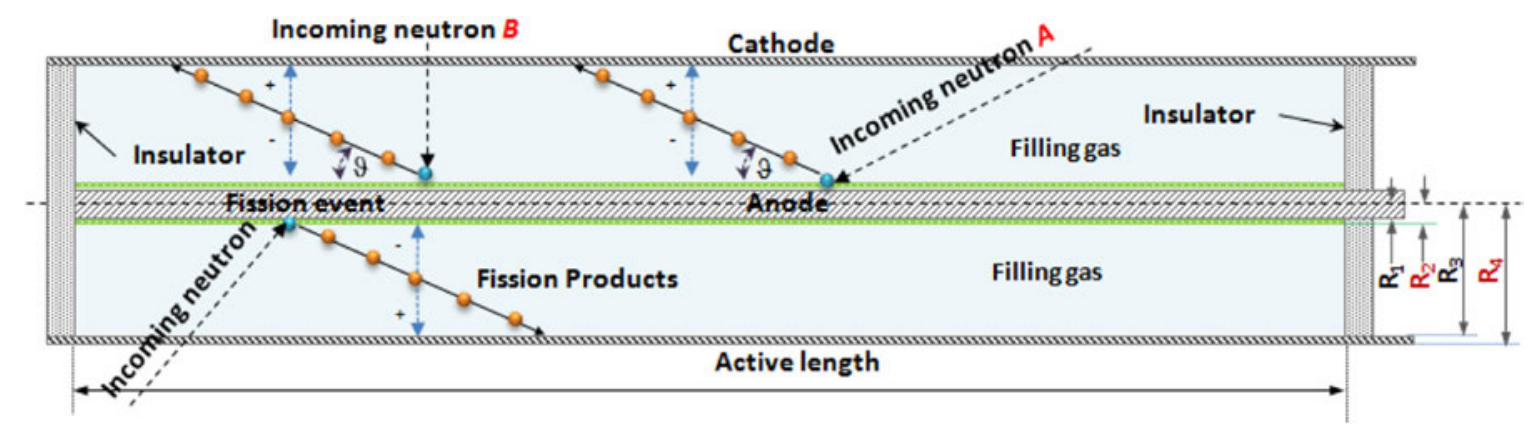

Fig. 1. General scheme of a fission chamber.

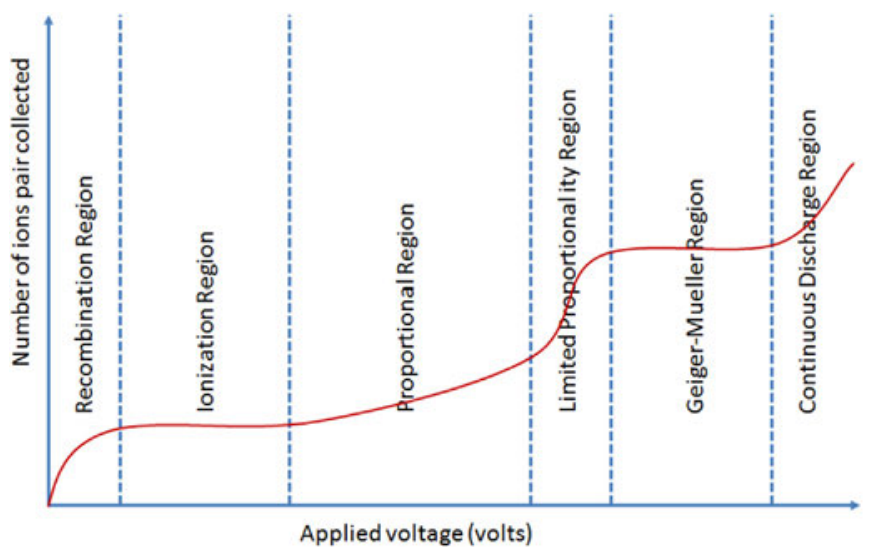

Fig. 2. Six-region curve for gas-filled detectors. Fission chambers are expected to work in the 'ionization region' or saturation plateau.

When a neutron interacts with an atom of the fissile material and produces fission, a couple of fission products are released resulting in an ionizing trajectory through the filling gas. A polarizing vol tage applied to the electrodes, prevents the ions and electrons from recombining. Electrons travel towards the anode and ions towards the cathode producing a pulse of current and potential. Ionization tracks have different angles and they produce different pulse shapes. Each neutron causing fission produces a pulse. All pulses occurring randomly in time add together. As neutron flux and hence pulse rate increases, accumulation occurs when pulses begin to coincide in time, and are counted as one by the electro nics connected to the chamber. Fig. 1 shows a schematic design of a fission chamber; in this picture a possible neutron absorber coating is also shown. This absorber could be use to filter the neutrons entering the chamber. Fig. 2 shows the six regions in which a gas filled detector can operate according to the polariza tion voltage applied:

I. Recombination region; in this region the charges produced by the passage of radiation quickly recombine to form neutral molecules due to the low voltage applied.

II. Ionization region; the collection efficiency of electron ion pairs in the recombination region increases with applied voltage until all the charges that are being produced get collected. In this region further increasing the voltage does not affect the measured current since all the charges being produced get collected by the electrodes. This zone is also called the "saturation plateau". Fission chambers work in this region.

III. Proportional region; if a high enough electric potential exists between the electrodes so that the charges could attain very high velocities, charges produced during primary ionization have enough energy that they can produce additional electron ion pairs, a process called secondary ionization.
IV. Limited proportional region; as the bias voltage increases, more charges are produced inside of the detector. Since heavy positive charges move much slower than the electrons, they form a cloud of positive charges between the electrodes. This cloud acts as a shield to the electric field and reduces the effective field seen by the charges. As a consequence the proportionality of the total number of charges produces to the initial number of charges is not guaranteed.

V. Geiger Mueller region; increasing the voltage further may increase the local electric field to such high values that an extremely severe avalanche occurs in the gas, producing very large number of charge pairs. Consequently a very large pulse of several volts is seen in the readout electronics.

VI. Continuous Discharge; if we continue increasing the bias voltage a breakdown process can occur, leading to a process of continuous discharge. In this region, electric arcs can be produced between the electrodes, which may eventually damage the detector.

Fission chambers can be operated in: pulse mode, current mode, and mean square voltage mode (MSV mode), also called Campbelling mode [3].

Several works have been carried out in order to qualify fission chambers in different nuclear facilities $[2,4$ 6] all of them have taken advantage of the existing tools to accomplish this task: MCNP, SRIM, GEANT4... using them at different stages of the design process. However this makes the design and analysis of sensitivity tedious and slow that is reason we have developed an integrated tool capable of dealing with the entire design and simulation process. As nuclear fusion facilities and new generation fission reactors are not in place yet, improvements in fission chambers performances will be needed. Thus, an integrated development tool for their design and outcomes simulation is of outstanding interest. During the last four years the present work has settled down the first stone for a software suit intended to give an integrated tool for the design of fission chambers capable of operating in such harsh environments Monte Carlo Fission Chamber Designer (MCFCD), which takes advantage of Monte Carlo methods. From the point of view of computing performance, Monte Carlo methods are generally easily parallelizable, with some techniques being ideal for use with large CPU clusters but presents higher CPU consumption. The results obtained from Monte Carlo calculations are based on probability decisions; hence the results obtained need a statistical analysis before taking a final decision. However Monte Carlo allows modeling the complete physical processes occurring in the fission chamber, thus it renders many intermediate data which can be very useful for a complete understanding of the device. Other efforts have been made in the fission chambers community in order to develop appropriate software for the design of these detectors using GARFIELD suite [4] or for the estimation of the $\gamma$ ray contribution to the signal [7]. 
MCFCD simulates the complete design cycle, from fission rate calculation to the electrical signal produced by neutron induced pulses. This makes easier the analysis of sensitivity when employing new materials, different nuclear data files, polarization voltages, etc. MCFCD has been conceived as a modular suit; it has been coupled with external data sources, such as SRIM or JANIS and includes an external database which contains the necessary data to perform the calculations for the complete design cycle and facilitate the addition and/or update of that data. As MCFCD simulates via Monte Carlo, the complete physical process taking place inside the chamber, many intermediate data is gathered, such as fission product inventory, distance traveled by fission products, absorptions occurring in the materials, etc. MCFCD also produces 3D coordinate's files that can easily be coupled with third parties plotting software (e.g. GNUplot) for ulterior study.

This work is organized as follows: next section explains the Monte Carlo Fission Chambers Designer code; in section three the numerical model implemented inside MCFCD is shown; section four introduces the results of the simulations performed; finally, main conclusions are shown in section five.

\section{Monte Carlo fission chamber designer}

MCFCD has been conceived as a decoupled software; simulator and database are two separate pieces of a single program. This architecture makes easier the integration of data from experi mental tests or new theoretical formulae in the simulation process saving computation time. It also permits the dynamic population of this database without having to rebuild the software. MCFCD is composed of three modules (see Fig. 3): the first one copes with radiation transport, the second one deals with pair production and the last one calculates the electrical signals. Additionally a shell script [8] has been developed in order to run MCFCD whilst simulating variable conditions; typically changing the bias voltage while plotting the electrical response curve. The database is the central piece of the model and precompiled data has been uploaded to it saving computation time.

MCFCD has the capability of using different data libraries, ENDF B VII.1 [9] being chosen in the present paper. Other important information, such as drift velocities of electrons and ions, has been collected from [10]. NEA evaluated libraries can be loaded fol lowing a straightforward procedure. The stopping power data is necessary for the calculation of the number of electrical carriers produced by the fission products; thus, SRIM [11] has been used since it has been qualified for the study the stopping power of fission fragments in gases [12]. An auxiliary module has been developed in order to produce sets of stopping power data and their ulterior upload to the MCFCD database. The calculation covers to the whole ENDF U235 fission yields library, ( 1247 fission products in total) and extends from 10 to $110 \mathrm{MeV}$, minimum and maximum emerging energy of U235 fission products. The gases employed to fill the inter electrode gap where, Ne20, Ar40, Kr83, and Xe131. More than 1.2 million rows of stopping power data for U235 have been uploaded to the database. MCFCD Database makes an easy task the addition of new materials and gases (pure or mixtures). Nevertheless in case of mixtures, especially organic gases, Penning effect must be taken into consideration $[13,14]$. The current database includes all the necessary information to com pute complete fission chamber simulations, from neutron trans port to the electrical signals.

MCFCD has been designed to perform 3D calculations. Thus dimensional parameters are provided to the simulator as volume boundaries. MCFCD produces output files containing 3D coordi nates allowing to easily plot neutron and fission products trajec tories in space. Those files of coordinates can be straightforward coupled with any plotting software (e.g. Gnuplot [15]). Fig. 4 shows the neutrons trajectories inside a generic chamber as taken from one of the MCFCD output files. Axis $\mathrm{x}, \mathrm{y}$ and $\mathrm{z}$, are the three dimensions of space, with the axis of the fission chamber located at $(0,0, z)$. 1st and 2 nd neutron sets represents the trajectories of two groups of neutrons taken from a simulation, the red grid represents the boundaries of the fission chamber.

MCFCD considers that neutrons are dimensionless points, neutron neutron interactions are neglected and neutrons travel in straight lines [6], collisions are instantaneous and point like and background material properties are isotropic, known and time independent. Fig. 5(a) shows the way in which MCFCD tracks neutron histories, it takes into account whether the neutron moves inside a cell or cross to another one. Cells are a MCFCD concept to define different materials and shapes that compose the fission chamber. Fig. 3(b) shows that MCFCD works with two kinds

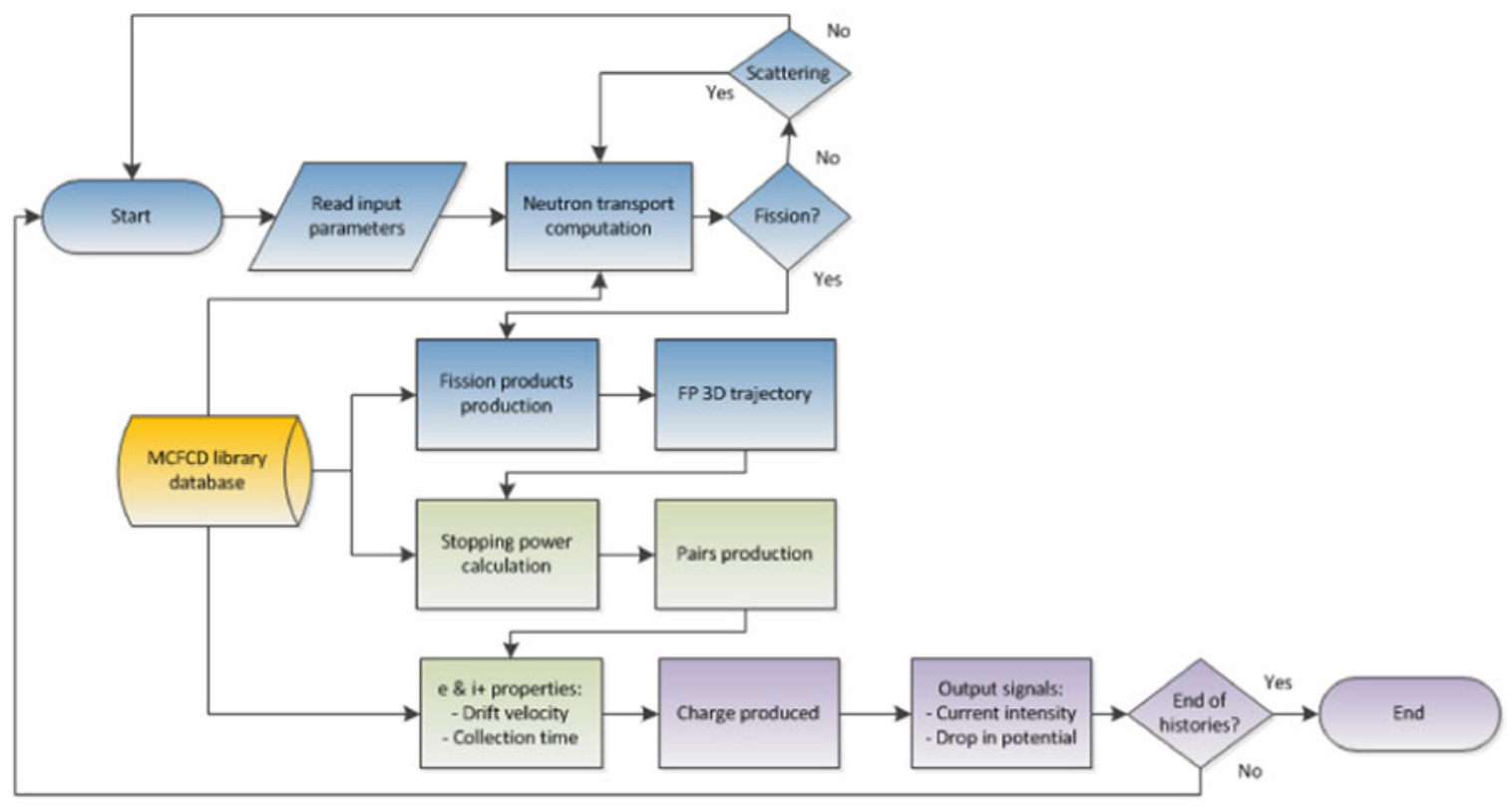

Fig. 3. High level flowchart of MCFCD simulation model. 


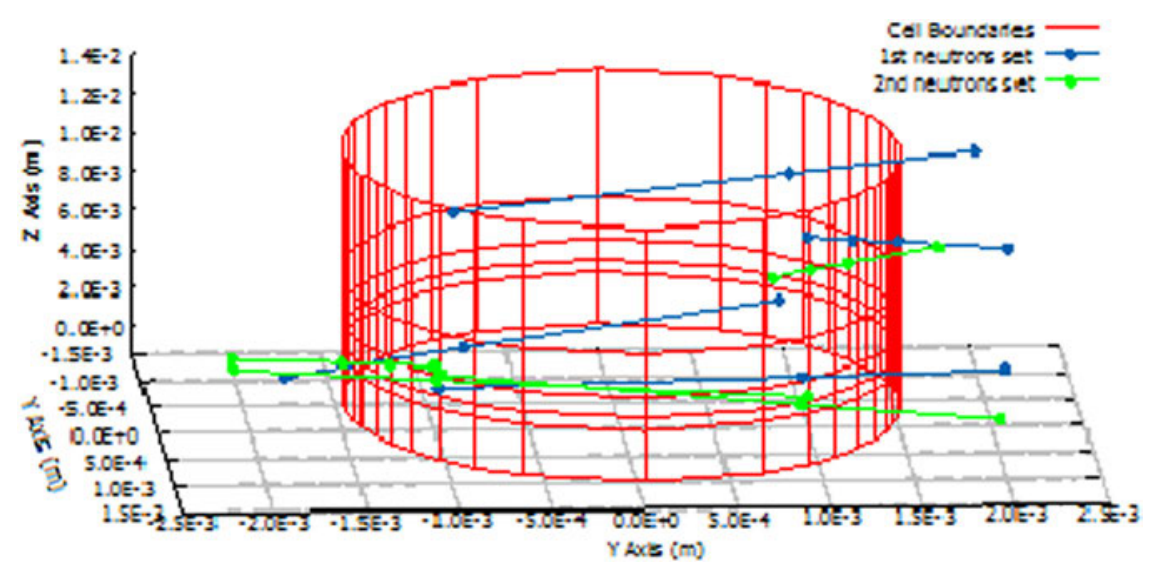

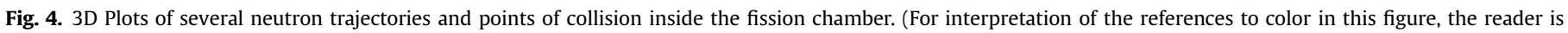
referred to the web version of this article.)

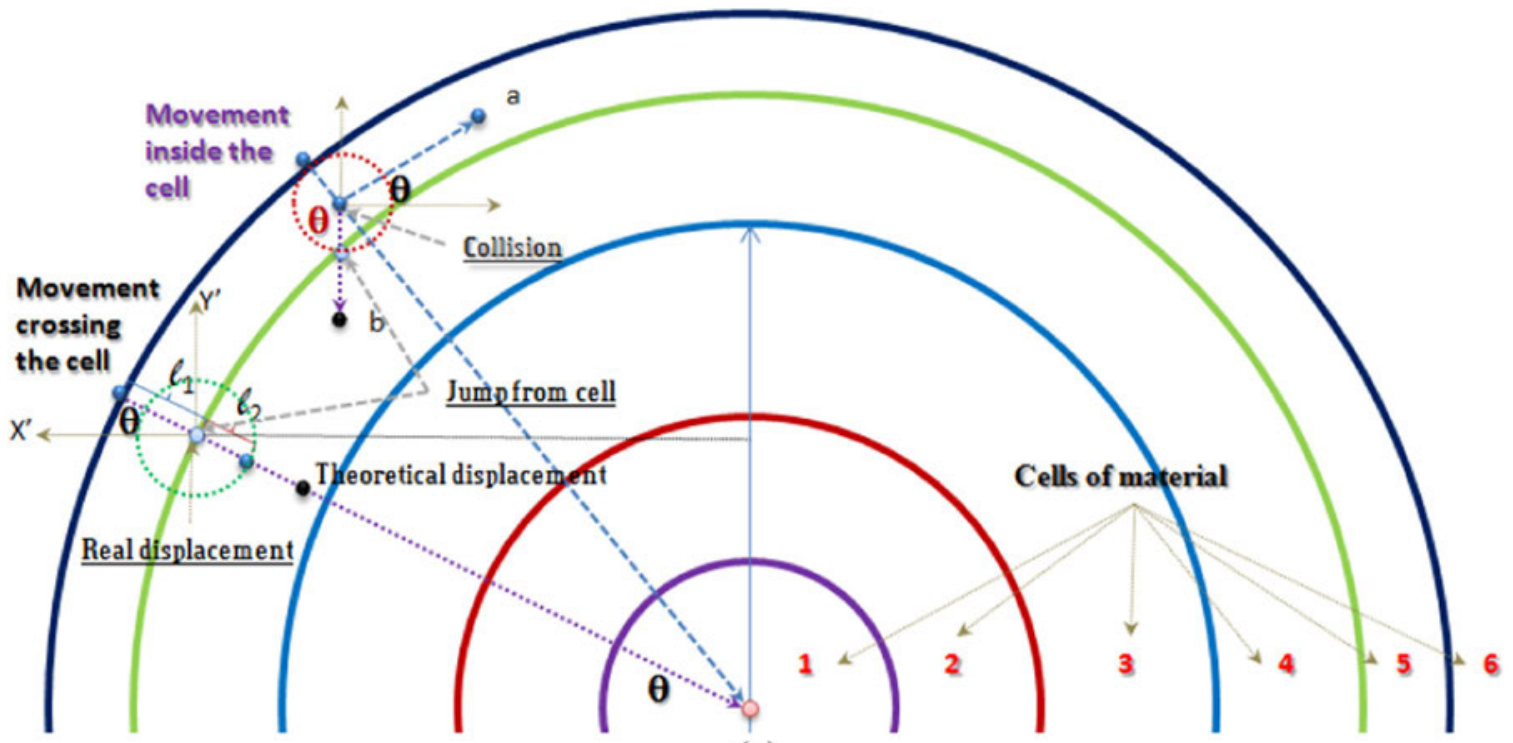

(a)

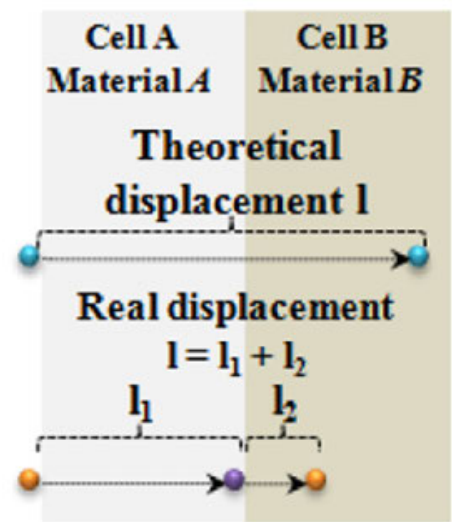

(b)

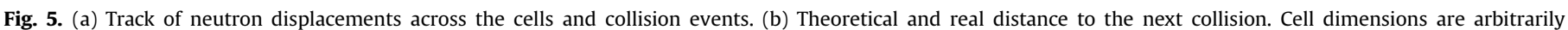
established.

of neutron displacements, one of them is considered theoretical, and the other one is considered the real displacement: when a neutron arrives at the frontiers of the cell, it is virtually stopped and, considering the properties of the following cell, MCFCD retrieves new material cross sections from the database and computes the distance the neutron is expected to penetrate into.
The real distance traveled is the sum of both displacements, $l_{1}$ corresponds to the distance to the border of the current cell; $l_{2}$ is the distance the neutron penetrates into the next one.

In order to simplify the calculations some hypotheses must be considered. Main assumptions have been taken from previous works and are listed below: 
An isotropic source of neutrons is assumed, following a trajec tory perpendicular to the longitudinal axe of the chamber.

The most important reactions in nuclear facilities are considered in MCFCD: $(n, f)$ fission, $(n, \gamma)$ radiative capture and $(n, n)$ elastic scattering.

Fissions produce two products and, due to their emerging energy is very high compared with the energy of the incident neutron, both are assumed to be emitted in opposite directions [13]. Thus, one is absorbed in the anode while the other one ionizes the gas.

Angles of emission of fission products are equally probable and, as shown in [16], self absorption on fissile coating is negligible. The simulated fission chamber has a cylindrical geometry having much larger length than diameter, thus electric field is assumed to be radial.

Space charge effects are not considered; hence fission fragments ionize the gas independently of the others.

The filling gas is homogeneous and does not evolve under irra diation [7].

The fission chamber is operated within the saturation region and the electric signal is considered to be mainly produced by neutrons; $\gamma$ rays contribution is neglected in this study [7]. External circuits, cable and so on, are not considered.

\section{Numerical model}

\subsection{Neutron transport}

MCFCD assumes that every neutron appears at the very edge of the outer chamber boundaries. In order to save computation cycles, it is considered that neutrons arrive at the chamber fol lowing perpendicular trajectories to the fission chamber axes; this guarantees that no neutron histories are lost because, at least in a first stage, all of them penetrate the boundaries of the chamber. This hypothesis introduces a bias which is explained below. After this first movement, dispersion angles are randomly calculated in spherical coordinates ( $\varphi$, azimuthal angle, $\theta$, polar angle, see Fig. 6) taking into account those most probable. Considering single and random events, $\left\{s_{i}\right\}, i=1, \ldots, n$, each of which is equally likely to occur whenever the random experiment is carried out, each of them has a probability of $1 / n$ [17]. Taking into account that MCFCD

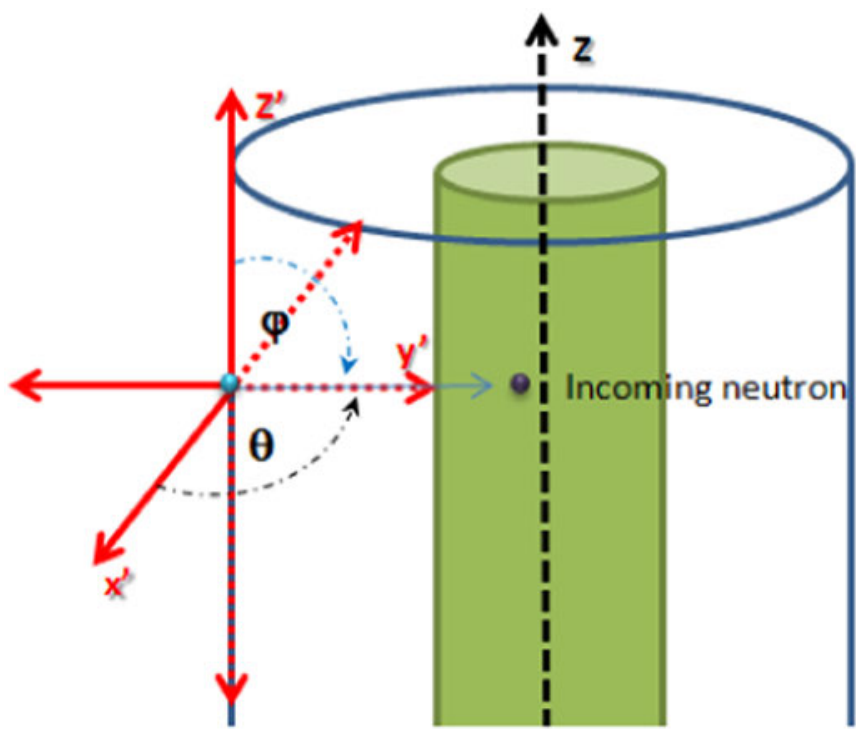

Fig. 6. MCFCD incoming neutrons are generated at the edge of the fission chamber and penetrate perpendicularly to its axis. samples one neutron at a time and $\varphi$ and $\theta$ are randomly sampled for every neutron history, hence the probability density function (pdf) of an incoming neutron to follow a trajectory with a specific $\varphi$ angle is $f_{2}(\varphi)=1 / 2 \pi$. In the same fashion, the pdf for an incoming neutron to arrive to the fission chamber with a specific angle $\theta$ is $f_{1}(\theta)=1 / \pi$. As these two are independent events, neutrons with trajectories perpendicular to the $z$ axis have a conditional pdf $f_{2 \mid 1}(\varphi \mid \theta)=f_{1,2}(\varphi, \theta) / f_{1}(\theta)=f_{1}(\theta) \mathrm{f}_{2}(\varphi) / f_{1}(\theta)=1 / 2 \pi$ [20]. This is the bias that MCFCD applies to the number of fissions.

The bias mentioned before does not affect the signal that the fission chamber produces, as it is explained later on, while heavily reduces the computation time. Since neutrons are neutral particles they do not carry any charge and therefore cannot interact in matter by means of the Coulomb force, which dominates the energy loss mechanisms for charged particles and electrons. This means that they do not produce ionization of matter for the mere fact of crossing it. When a neutron does undergo interaction, it is with a nucleus of the absorbing material.

Therefore, in the production of the electrical signal of a fission chamber the neutrons that matter most are those who produce fissions (in this work is only considered the signal produced by neutrons), independently of their flight paths. Fig. 1 shows two neutrons, $A$ and $B$, having different inbound trajectories; neutron $B$ perpendicular to the longitudinal axis of the fission chamber and neutron A having an arbitrary incoming angle and hence a longer flight path. As the emerging angle of fission products is a random event, it can be considered the circumstance in which the emer ging angle $\vartheta$ of both fission products is the same. Under those premises it can be observed that the path covered by both fission products is the same. Hence they produce the same number of electron ion pairs and therefore the same electrical signal, inde pendently of their respective incoming angle.

Neutrons penetrate into the fission chamber following a straight line until they collide $[18,19]$. The probability that a neu tron has a collision at a distance $\mathrm{s}$ along the flight path is:

$P(l)=\sum_{t}(l) \exp \left[\int_{0}^{l} \sum_{t}\left(l^{\prime}\right) d l^{\prime}\right]$

where $\sum_{t}$ is the total macroscopic cross section of the material; (1) is the probability distribution function for the collision distance $l$; which has a cumulative distribution function:

$\int_{0}^{l} P\left(l^{\prime}\right) d l^{\prime}=1 \quad e^{-\sum_{t} l}$

By setting the cumulative distribution function equal to a random number $\xi$, on the interval $[0,1)$, and solving for the dis tance $l$, the distance to next collision can be sampled as:

$l=\frac{\ln (1 \xi)}{\sum_{t}}$

Since $\xi$ is uniformly distributed on $[0,1)$, it implies that (1 $\xi$ ) is uniformly distributed on $[0,1)$ as well. Hence, the distance to the next collision is:

$l=\frac{\ln (\xi)}{\sum_{t}}$

The occurrence of a collision is a rare event, and even less frequent is the occurrence of fissions, thus MCFCD applies biasing techniques in order to increase the probability of a collision to occur within the distance of control; which in our case corre sponds to the thickness of the fissile material. According to (4), the distance a neutron travel to the next collision is proportional to the inverse of the total cross section $\sum_{t}$. Taking into account the properties of the probability, it is possible to act on this parameter artificially increasing it to force neutrons to travel shorter dis tances inside the fissile coating and hence increasing the 
probability of experimenting collisions. Therefore, the probability of achieving fissions is higher. Taking an arbitrary scalar $N \in \mathbb{R}, \Sigma_{t}^{\prime}=N \Sigma_{t}^{\prime}$, one can obtain:

$l^{\prime}=\frac{\ln (\xi)}{\Sigma_{t}^{\prime}}$

Where $l^{\prime}$ is $N$ times smaller than the dimension retrieved from (4). At the end of the simulation process one can revert the bias introduced by $N$, by means of dividing the number of fissions and captures achieved, by $N$.

When a collision occurs at a distance $l$ into cell $n$, it is necessary to determine what type of nuclide and what type of reaction occurred. The probability of a reaction $x$ with a nuclide of specie $i$ is defined by:

$P_{i x}=\frac{N_{i} \sigma_{i x}}{\sum_{i, x} N_{i} \sigma_{i x}}$

where $N_{i}$ is the number density of nuclide $i$ in region $n, \sigma_{i x}$ is the microscopic cross section for reaction $x$ for nuclide $i$ at the energy of the neutron. A homogeneous material is considered, therefore the probability of capture is determined by $P(c)=\sum_{c} / \sum_{t}$, elastic scattering $P(s)=\sum_{s} / \sum_{t}$ and fission $P(f)=\sum_{f} / \sum_{t}$, where $\sum_{t}$ stands for the macroscopic total cross section. Hence selecting a CDF, $0 \leq F(s) \leq 1[21]$ and setting the CDF equal to a random number $\zeta$ on the unit interval, capture will occur when $\zeta_{1} \leq \sum_{c} / \sum_{t}$, scat tering if $\sum_{c} / \sum_{t} \leq \zeta_{2} \leq \sum_{s} / \sum_{t}$ and fission in case $\zeta_{3}>\sum_{s} / \sum_{t}$.

Consider a neutron moving with energy $E_{L}=\frac{1}{2} m v_{L}^{2}$ in the laboratory systems $(L)$ incident upon a stationary nucleus of mass $M$. Since only the relative masses are important in the kinematics, $m=1$ and $M=A$ (mass number); taking into consideration the velocity of the center of mass CM system in the $L$ and considering that the speeds of the neutron and the nucleus in the CM system do not change during the scattering event, one can obtain a rela tionship between the incident and final energies of the neutron in the $L$ system and the scattering angle in the CM system [21]:

$E_{L}^{\prime}=E_{L} \frac{A^{2}+1+2 A \cos \theta_{c}}{(A+1)^{2}}$

Assuming the emission of the outgoing particle is azimuthally symmetric (emission is equally probably into a cone subtended by the scattering angle $\theta_{c}$ ); the outcome of the collision is completely determined if we specify another degree of freedom; the particle energy $E_{L}$ or $\theta_{c}$. Eq. (5) determines the emerging energy of the neutron after collision; thus we choose the angle $\theta_{c}$. In case of isotropic scattering, as it happens in CM system for high energy neutrons and heavy nucleus, dispersion is independent of the angle. Thus $\theta_{c}$ can be defined as:

$\theta_{c}=\operatorname{ar} \cos (1 \quad 2 \cdot R N 1) ; 0 \leq \theta_{c} \leq \pi$

Where RN1 is a random number of the unitary interval $0 \leq \mathrm{RN} 1 \leq 1$

Generating another random number and equating it to the CDF for $\varphi$ the direction of the scattered neutron can be determined. The calculation is repeated for the scattered neutron until the neutron either leaks from the system or it is absorbed or produces fission. Fission and absorption are considered the end of a neutron history. Azimuthal angle is equally probable in the interval $[0,2 \pi]$, thus $0 \leq \varphi \leq 2 \pi$; hence $\varphi=\mathrm{RN} 2 \cdot 2 \pi$; where RN2 is another ran dom number of the unitary interval $0 \leq \mathrm{RN} 2 \leq 1$. The total kinetic energy of any fission fragment can be estimated assuming that the initial kinetic energy of a fragment is inversely proportional to their masses, and using an empirically defined dependence of the average kinetic energy of fission fragments on the mass and the charge of a fissioning nucleus [22].
Table 1

Example of fission products inventory reported.

\begin{tabular}{llllllll}
\hline FP & Kr89 & Cs142 & Zr102 & Ba144 & Zr97 & Zr103 & I135 \\
Compl. FP & Ba144 & Rb90m & Te131m & Kr89 & Te136 & Te129m & Y98m \\
\hline
\end{tabular}

\subsection{Fission fragments}

Gaseous fission products are of outstanding importance, since the mixture of small quantities of alien products with the filling gas can strongly slope up or down pair formation, alter the size of the saturation plateau [23] and influence the drift velocity of electrical carriers. MCFCD uses Monte Carlo and fission yield libraries to provide fission products inventory. Table 1 gives an example of fission products inventory.

\subsection{Pairs production}

Ionization is more intense during the final section of the dis placement of an ionization particle because a slower velocity enables a higher probability of interaction between particle and atoms of the gas [24]. However due to the dimensions of a fission chamber it is acceptable to consider constant ionization along the flight path of the fission product. MCFCD tracks trajectories of fission products independently, thus it can determine the dis tances $\Delta x$ traveled by them. Knowing these distances and the stopping power computed in advance with SRIM, together with the $W$ value (the average energy needed to create an electron ion pair in a gas) of the filling gas, the number of pairs is determined by:

$N=\frac{1}{W} \frac{d E}{d x} \Delta x$

where $d E / d x$ represents the stopping power. MCFCD operates in the saturation zone; hence solely primary ionization occurs. For this reason, only one $\mathrm{e}^{-}$and one ion ${ }^{+}$come out from every pair. In order to simplify calculations without losing accuracy, the idea of a "center of moving charges", taken as the centroid of the fission product trajectory, is introduced. A similar argument is exposed in [23]. This hypothesis is based on two pillars: firstly, the reduced dimensions of a fission chamber and the high drift velocities of electrons and ions ensures collecting times are always going to be in the same order of magnitude independently of the position in the inter electrode gap. Secondly, considering linear pairs pro duction, these are going to appear as soon as the fission fragment departs from the fissile deposit; the electrons will be collected virtually instantaneously while the ions must cross the entire gap. The opposite will happen when fission fragments reach the cath ode. Thus it is possible to settle an average drift velocity and col lection time for both carriers and hence estimate the contribution of each particle to the output signal. Fig. 7 shows the model explained before.

Fig. 8 plots data gathered from SRIM simulations in which one can see the evolution of the stopping power of one of the typical U235 fission products versus gas pressure. A linear behavior is observed with the gas pressure.

When electrons and ions are released under the influence of an applied electric field, one of them moves apart from the other. As these carriers move in the heart of an electric field, they induce an electric pulse that can be measured. When electrons are released they immediately begin to drift in opposite direction to that of the electric field, ions move much more slowly than electrons, usually $10^{3}$ times slower, towards the cathode. Electron and ions mobi lities $\left(\mu_{e}, \mu_{i}\right)$ and drift velocities have been compiled from [10].

In gaseous detectors the electrical charge, change in potential and current intensity, depends not only on the electrons; the ions 


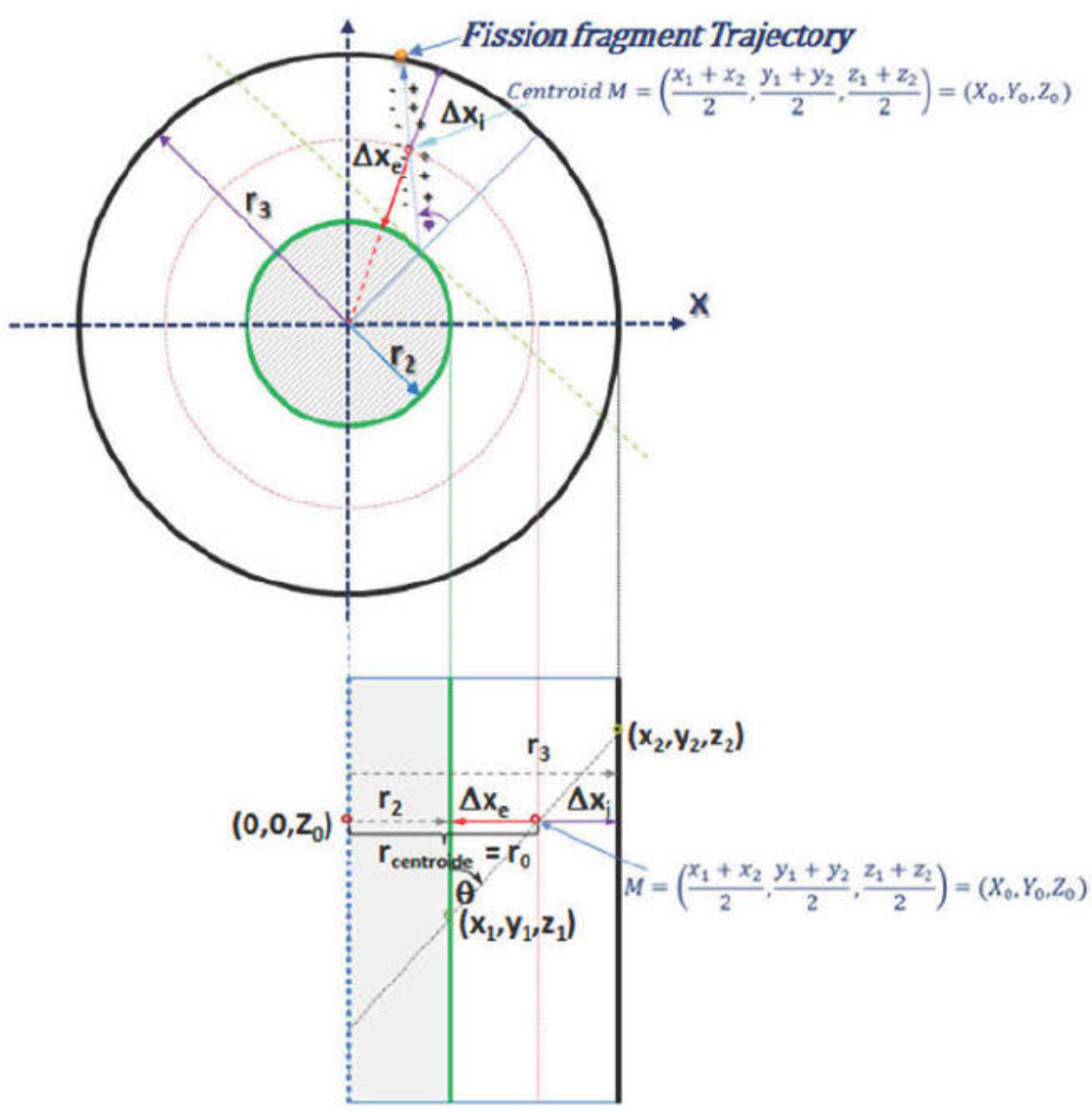

Fig. 7. Representation of electron and ion drift velocities and collection time calculation. A generic fission fragment trajectory is shown.

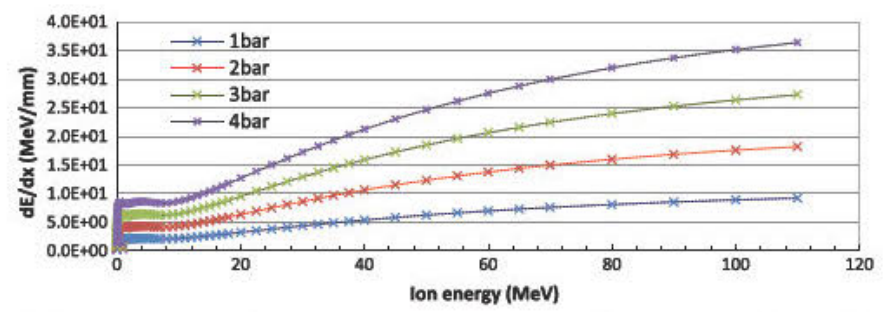

Fig. 8. Evolution of stopping power vs gas pressure. Data gathered from SRIM simulations.

contribute as well. In the presence of an electric field, ions move towards the negative electrode with a velocity known as the drift velocity, $v_{d}$. It has been found that it is proportional to the ratio of electric field and gas pressure [24].

$v_{d}=\mu_{+} \frac{E}{p}$

$E$ represents the electric field, $p$ is the pressure of the gas and $\mu_{+}$is the ion mobility. Mobility depends on the ions mean free path inside the gas, the collision with gas atoms and the energy distribution [24].

During fission chamber operation the electrons acquire a greater acceleration than that of the ions because of their much lower mass. Electrons do not move in a straight line along the trajectory, due to encounters with neutral gas atoms or molecules, its motion is complicated and zigzagged. However electrons will try to move along the lines of force of the electric field and, as in a cylindrical geometry, those lines are radial and the collision events are independent one from the other, the resultant motion could be consider as a straight line. Electron velocities are much higher than that of the ions and collection times much shorter, typically $\approx 10{ }^{9} \mathrm{~s}$. When both carriers are released, electrons immediately start to drift towards the anode and due to much shorter collection time, when they arrive to the electrode, ions have barely moved from their original position [25]. Due to the high velocity of elec trons and reduced dimensions of the chamber can be considered electrons have a constant velocity. Thus based on the model shown in Fig. 7, and assuming drift velocity of electrical carriers is constant inside the fission chamber due to the tiny dimensions, one can estimate the drift time as $t_{i}=\Delta x_{i} / \nu_{i}$ and $t_{e}=\Delta x_{e} / \nu_{e}$.

\subsection{Electrical signal}

The electrical signal produced by electrical carriers can be measured in two ways, via a drop in potential or through a signal of intensity. The total change in potential expected due to the movement of electrons and ions in a cylindrical chamber, having a total charge $Q,\left(N_{\text {tot }} \cdot e\right.$ ) can be computed as [24]:

$V=\frac{Q}{2 \pi \epsilon l} \ln \left[\frac{b}{a}\right]$

Here $\varepsilon$ represents the gas permeability and $l$ the chamber length, $b$ and $a$ are the outer and inner radius. Eq. (7) shows that for a cylindrical chamber the pulse height is independent of the point in which the carriers were produced.

The current delivered by a fission chamber is not a constant signal; it is constituted of sorts of pulses appearing randomly as fission events occur. For a fission chamber operating in Current Mode and within the saturation region, the average current is the product of the event rate and the charge produced per event [3]:

$I=\bar{r} \bar{q}=\Phi_{t h} \chi \bar{q}$ 


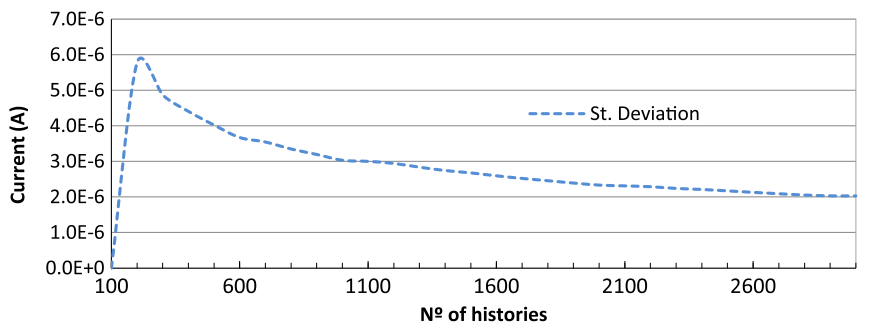

(a)

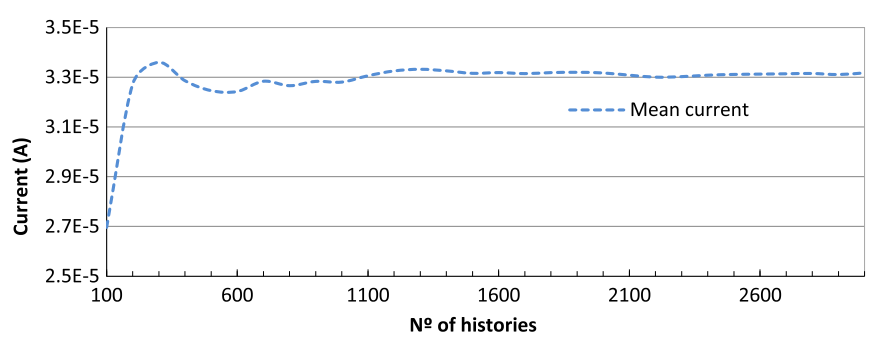

(b)

Fig. 9. (a) Standard deviation showing the statistical error and (b) convergence to the mean current of the simulated current signal.

Where $r=$ event rate $(f / s), \bar{q}$ is the charge per event. It stands for the amount of electrical carriers produced per fission product and the charge produced per carrier, $q=1.6 \cdot 10{ }^{19} \mathrm{C}, \Phi_{\text {th }}=$ thermal flux ( $\mathrm{n} \mathrm{cm}^{2} \mathrm{~s}^{1}$ ), $\chi=$ the fission efficiency (fissions/neutron). Thus, (8) leads to

$I=\chi \bar{\Psi} \Phi_{t h} S q$

where $\bar{\Psi}$ represents the electron ion pairs produced per fission. Fig. 9(a) shows that the signals calculated with MCFCD have a statistical error which decreases by $1 / \sqrt{ } n^{\circ}$ histories. Fig. 9(b) also shows that $10^{3}$ histories are enough for a smoothly convergence to the solution.

The charge collection efficiency is defined as $f=Q^{\prime} / Q$ by Boag's Treatment of Mie's Theory of General or Volume Recombination [26], and corresponds to the effective charge collected versus total charge produced inside the chamber. This parameter is really important in order to evaluate the regime in which the chamber is operating. As suggested in [26], and taking into consideration the parameters of our fission chamber model, the continuous radia tion theory of ionic recombination can be used, under the pre mises of having fission rates in the order of $10^{8}$ or $10^{9} \mathrm{f} / \mathrm{s}$ what represents a pulse occurs every $10^{8}$ or $10^{9}$ seconds on average, which means many pulses occur during ion transit time. In that case charge collection efficiency could be represented as:

$f=\frac{1}{1+\frac{1}{6} f^{2}}$

where

$f^{2}=\frac{\alpha}{e k_{1} k_{2}} \frac{P}{P_{0}} \frac{d^{4} q}{V^{2}}$

Here $f$ is a dimensionless parameter which depends on the chamber geometry [26 27], $e$ is the electron charge, $k_{1}, k_{2}$ electron and ion mobilities, $\alpha$ the first Townsend recombination coefficient, $q$ is the ionization charge density, $P_{0}, P$ are gas at STP and operation conditions respectively, $V$ voltage applied and $d$ the equivalent electrode separation form cylindrical geometry[26]. Eq. (10) allows plotting the saturation curve for recombination and ionization regions. Charge collection efficiency allows finding the polariza tion voltage representing the beginning of the ionization zone and hence the region in which the chamber operates in saturation. It is generally accepted that saturation plateau begins when $f \geq 0.96$ [26]. No general bias voltage can be pre established for the

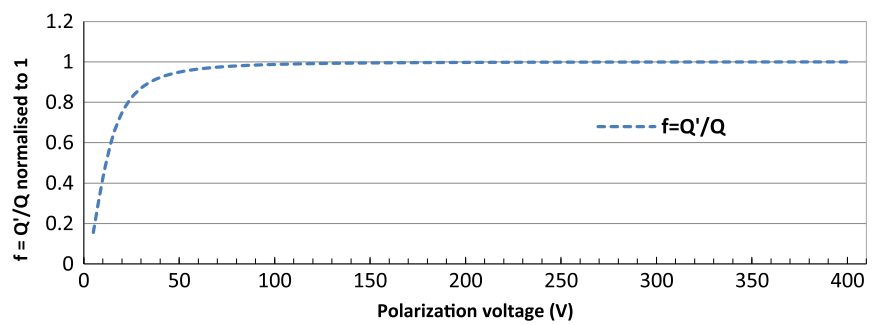

Fig. 10. Charge-collection efficiency curve versus polarization voltage.

beginning of saturation since threshold limits depend on the geometry, materials and the operational conditions. In this case the charge collection efficiency curve is illustrated in Fig. 10.

\section{Results of numerical simulations}

In this section a set of data outcome from different simulation is presented, showing the capabilities of the MCFCD software. The dimensions of a generic fission chamber are given in order to compare the MCFCD simulated data with other already published.

Table 2 exhibits the most important parameters of the chamber considered for the present study, as well as the characteristics of a well known comercial detector, the CFUR43 by PHOTONIS [28]. CFUR43 dimensions were collected from Refs. [7,13,30].

The fission rate spectrum showed in Fig. 11(a) has been obtained when simulating 242 groups of energy of a generic thermal neutron spectrum, as that showed in [29], the fission chamber data from Table 2 and ENDF B VII.1 library for U235. As theoretically expected, fission rate is maximum in the thermal region $(0.0250 .1 \mathrm{eV})$, it is reduced in the epithermal region $(0.1$ $0.4 \mathrm{eV}$ ), clear definition of the resonance energy range (10 to $300 \mathrm{eV})$, with a pick on the $1 \mathrm{eV}$. Fission rate increases in the fast neutrons region, $(\approx 1 \mathrm{MeV})$, because ENDF B VII.1 cross sections rises between approximately 1 and $8 \mathrm{MeV}$. Plot of MCFCD simu lated fission efficiency (fissions/neutron) showed at Fig. 11 (b) faithfully reproduces the ENDF B VII.1 fission cross section shape.

MCFCD informs fission rates between $3.87 \cdot 10^{8} \mathrm{f} / \mathrm{s}$ for fluxes of $3.4 \cdot 10^{12} \mathrm{n} \mathrm{cm}^{2} \mathrm{~s}^{1}$ and $3.85 \cdot 10^{9} \mathrm{f} / \mathrm{s}$ for fluxes of $3.4 \cdot 10^{13} \mathrm{n} \mathrm{cm}^{2} \mathrm{~s}^{1}$ which are in the same range of those repor ted in [23] for a fission chamber of similar characteristics.

As explained in Section 2, MCFCD implements a Monte Carlo simulation module for fission products inventory. This module must accurately match fission product distribution according to theoretical data provided by fission yield libraries. Fig. 12 confronts the thermal U235 ENDF B VII.1 independent fission yields (blue

Table 2

Geometrical and physical features of the fission chambers considered for testing MCFCD results.

\begin{tabular}{|c|c|c|c|}
\hline & Unit & MCFCD design & CFUR43 \\
\hline$r_{a}$ (Anode outer radius) & $\mathrm{m}$ & $9.93 \mathrm{E}-4$ & $1 \mathrm{E}-3$ \\
\hline $\begin{array}{l}r_{x} \text { (Cathode inner radius }+ \text { fissile } \\
\quad \text { coating) }\end{array}$ & $\mathrm{m}$ & $1 \mathrm{E}-3$ & - \\
\hline$r_{b}$ (Cathode inner radius) & $\mathrm{m}$ & $1.25 \mathrm{E}-3$ & $1.25 \mathrm{E}-3$ \\
\hline$r_{c}$ (Cathode outer radius) & $\mathrm{m}$ & $1.50 \mathrm{E}-3$ & $1.50 \mathrm{E}-3$ \\
\hline$l$ (Sensitive length) & $\mathrm{m}$ & $13 E-3$ & $13 \mathrm{E}-3$ \\
\hline Fissile layer thickness & $\mathrm{m}$ & $7 E-6$ & - \\
\hline Fissile layer volume & $\mathrm{m}^{3}$ & $5.7 \mathrm{E}-10$ & - \\
\hline$\mu_{s}$ Fissile layer (areal density) & $\mathrm{mg} \mathrm{cm}{ }^{2}$ & - & 200 \\
\hline$\mu_{v}$ Fissile layer (volumetric density) & $\mathrm{kg} \mathrm{m}^{3}$ & $19.050 \mathrm{E}+3$ & - \\
\hline Mass $\mathrm{U}^{235}$ & $\mu \mathrm{g}$ & 163.36 & - \\
\hline Filling gas & - & $\mathrm{Ar}^{40}$ & $\mathrm{Ar}$ \\
\hline Gas pressure & bar & $1.1-4$ & $1.1-4$ \\
\hline
\end{tabular}




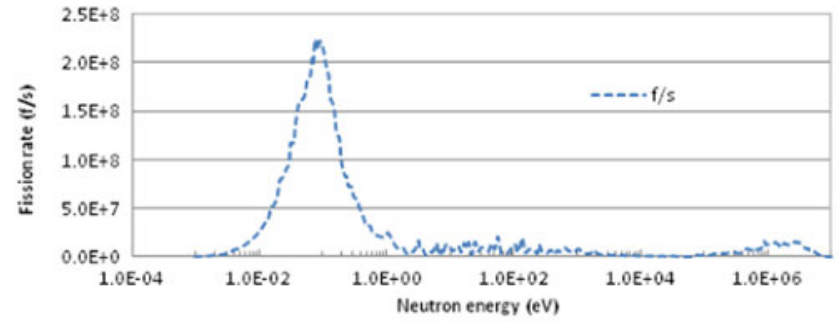

(a)

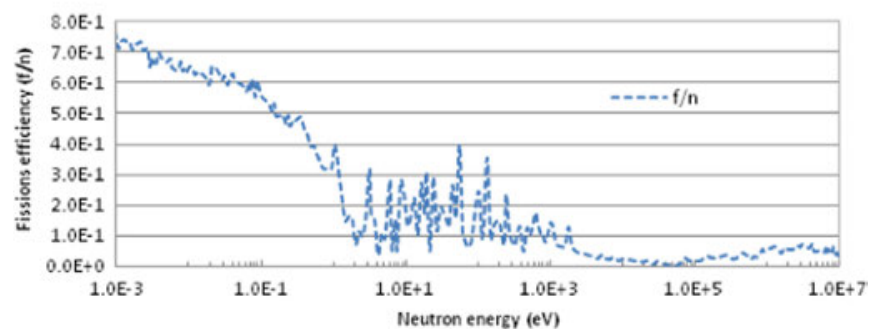

(b)

Fig. 11. MCFCD fission rate (a) and fission efficiency (b) simulation vs neutron energy.

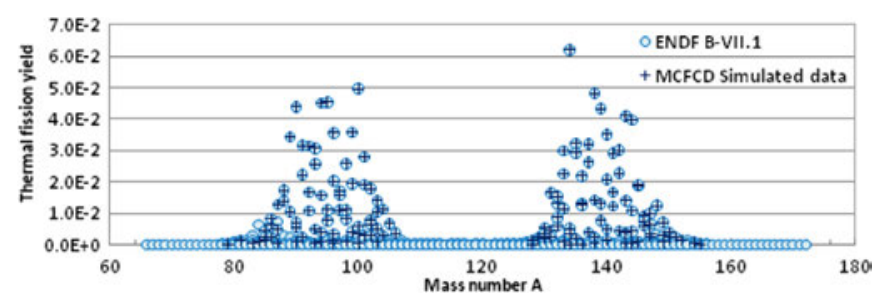

Fig. 12. ENDF B-VII.1 $\mathrm{U}^{235}$ independent fission product yields versus simulated data. (For interpretation of the references to color in this figure, the reader is referred to the web version of this article.)

Table 3

Comparison between electron-ion pairs calculated through MCFCD with those reported in [23] and [30], per unit of pressure and distance.

\begin{tabular}{llll}
\hline & Chabod [24] & ICRU Report 31 [31] & MCFCD Simulation \\
\hline Pairs P m $^{1}$ & $1.8 \cdot 10^{8}$ & $2 \cdot 10^{8}$ & $2.1 \cdot 10^{8}$ \\
\hline
\end{tabular}

circles) with MCFCD simulations (black cross), showing that simulated data correctly match with ENDF B VII.1 library.

According to Eq. (6), electron ion pairs production heavily depends in two parameters: the stopping power and the distance covered by the fission product, (assuming linear loss of energy). MCFCD computes the distance covered by each fission product giving an average figure at the end of the simulation process. Table 3 compares MCFCD electron ion pairs production data with those reported in [23], as well as those recommended in [30].

Electron ion pairs simulated by MCFCD are $4.8 \%$ higher than those reported by [30], and $14 \%$ than [23]. It is worth noting that the figures given in [23] and [30] are averaged figures for generic trajectories, while MCFCD simulates 3D paths one by one. There fore, the value simulated with MCFCD should be more accurate.

Fig. 13(a) and (b) show pulses of current and potential obtained with MCFCD. Those occurring randomly combine to give an aver age DC or potential signal that can be used to estimate the neutron flux. The pulses amplitude could be used as the neutrons footprint.

MCFCD mathematical model treats separately drift of electrons and ions through the gas. As a result of this, it allows analyzing the contribution of both carriers to the current signal. In agreement with theory [31 32], MCFCD reports that ions contribution could be considered negligible in comparison with the one of electrons; the current signal of electrons being about $10^{3}$ times bigger.

In order to show the response of the fission chamber shown in Table 2, the evolution of the mean current signal has been depicted in Fig. 14 for different gas pressures. It has been estimated for a fix thermal flux, $\Phi_{t h}=3.4 \cdot 10^{12} \mathrm{n} \mathrm{cm}^{2} \mathrm{~s}{ }^{1}$. The beginning of the saturation plateau, chosen as a charge collection efficiency $\mathrm{f} \geq 99 \%$, is also shown. As expected the saturation current for 4 bars of pressure is about 4 times bigger than the one for 1.1 bars. One can also see that the saturation plateau begins at lower vol tages when the pressure decreases. Thus for a given neutron flux,

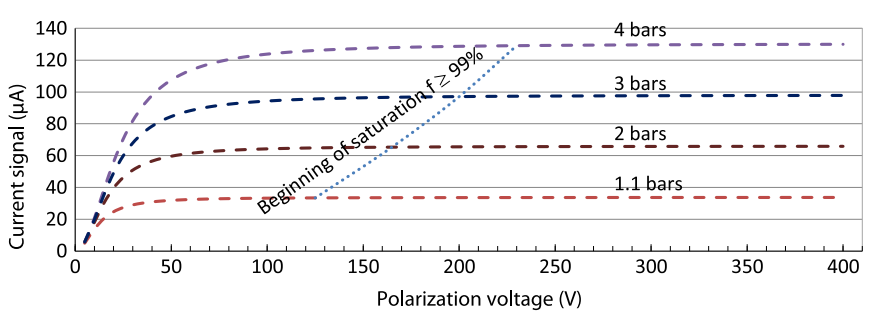

Fig. 14. Mean current signal vs polarization voltage for various gas pressures, $\Phi_{\text {th }} 3.4 \cdot 10^{12} \mathrm{n} \mathrm{cm}^{2} \mathrm{~s}^{1}$.

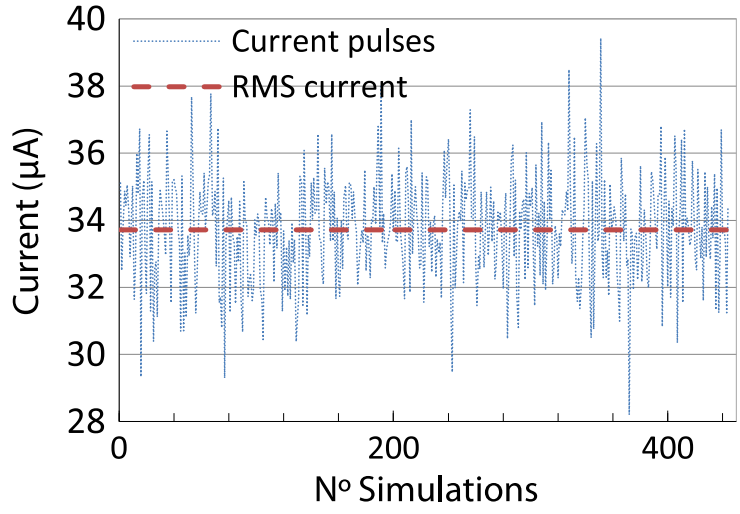

(a)

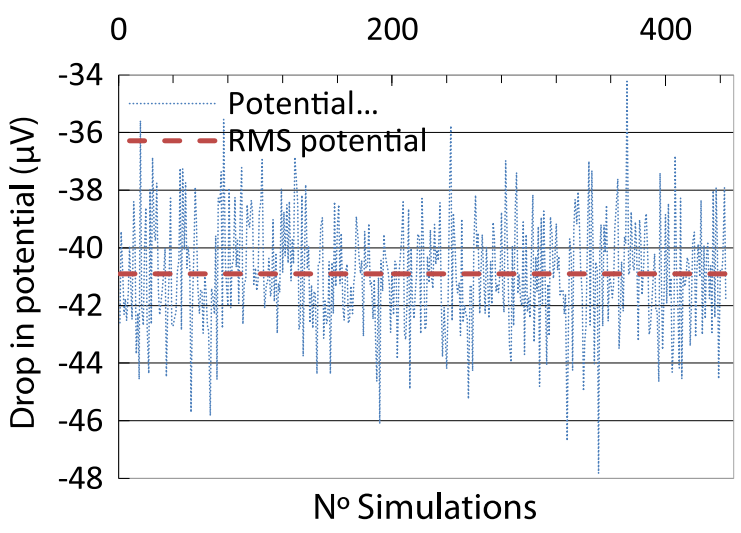

(b)

Fig. 13. MCFCD simulation of (a) pulses of current and (b) potential and respective RMS signals. 


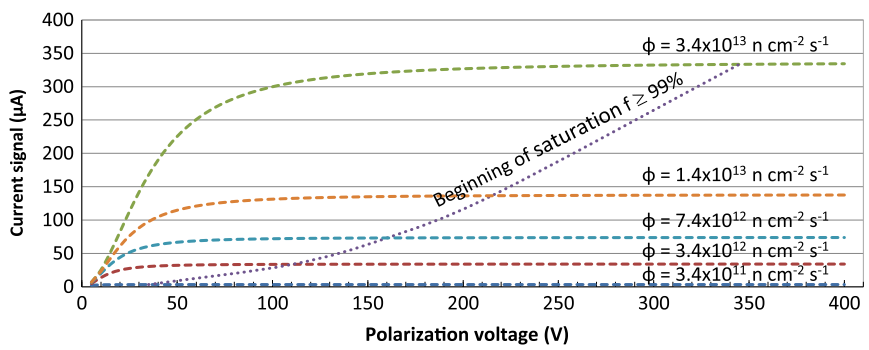

Fig. 15. Current signal of a 1.1 bar fission chamber and various neutron fluxes.

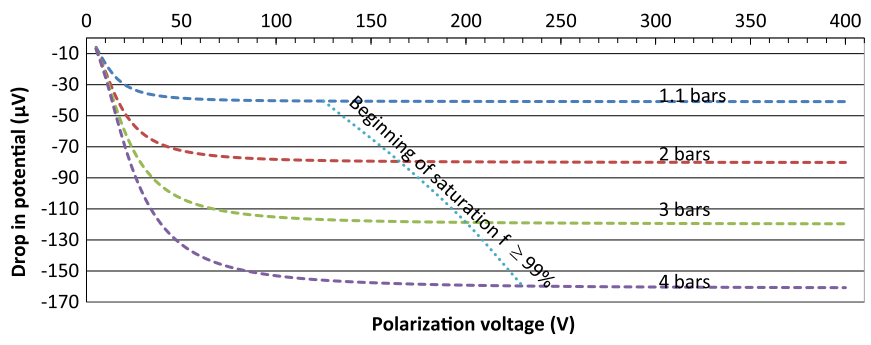

Fig. 16. Mean drop in potential vs polarization voltage for various gas pressure and $\Phi_{\text {th }} 3.4 \cdot 10^{12} \mathrm{n} \mathrm{cm}^{2} \mathrm{~s}^{1}$.

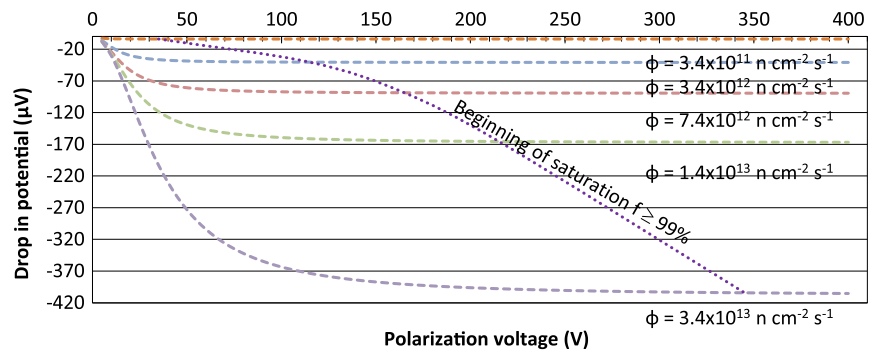

Fig. 17. Drop in potential of a 1.1 bar chamber for various neutron fluxes.

we can obtain saturation at lower voltages reducing gas pressure, however the current signal will be penalized. This rule is correctly simulated by MCFCD. MCFCD reports a saturation voltage of $125 \mathrm{~V}$ for 1.1 bar fission chamber, which is close to the $150 \mathrm{~V}$ that [28] recommends as operation voltage for its CFUR43 model; fission chamber used as reference in this paper. Fig. 15 shows the output current delivered by the fission chamber considered in Table 2 when it is exposed to different neutron fluxes; it shows the polarization voltage to which the saturation plateau begins as well. Thus in real operation one could estimate the existing neutron flux from output signal.

MCFCD provides two ways of measuring neutron fluxes, either via current intensity or through drop in potential. Fig. 16 repro duces the drop in potential when the chamber operates at differ ent gas pressures while Fig. 17 reproduces the drop in potential as a function of the neutron flux.

Finally, a comparison of the chamber sensitivity with that reported in [13] has been performed, in order to check the
Table 5

Comparison of intensities of saturation for 1.1 and 4.0 bar fission chambers.

\begin{tabular}{lllll}
\hline$I_{\text {sat }}(\mu \mathrm{A})$ & 1.1 bar & Difference (\%) & 4 bar & Difference (\%) \\
\hline$I_{\text {sat measured }}[13]$ & 37 & & 120 & \\
$I_{\text {sat MCFCD }}$ & 33.4 & 9.7 & 129 & 7.0 \\
\hline
\end{tabular}

performance of MCFCD. Results have been collected in Table 4 for different pressures and a thermal neutron flux $\varphi_{\text {th }}=3.4 \cdot 10^{12} \mathrm{n} \mathrm{cm}^{2} \mathrm{~s}{ }^{1}$. It is worth noticing that MCFCD data is in good agreement with the one measured in [13]. The Diff. col umn represents the difference between measured sensitivities calculated by [13] and simulated by MCFCD, highlighting the good agreement.

Table 5 compares saturation intensities from [13] with those of MCFCD for 1.1 and 4.0 fission chambers. Data reported by MCFCD highlight a good agreement with those measured by [13].

\section{Conclusions}

An integrated software suite named MCFCD has been devel oped for the design of fission chambers. It provides different simulation capabilities, such as fission rate calculation, fission products produced, mean free path of fission products, mean free path of electrons and ions, output current, drop in potential, saturation intensity and chamber sensitivity. It also produces 3D coordinates for neutrons and fission product trajectories.

The results obtained with MCFCD are in good agreement with technical data such as those recommended by Photonis [28] for CFUR43 and with experimental data as those informed in [13] and with other theoretical models such as $[13,23]$. MCFCD reports a saturation voltage of $125 \mathrm{~V}$ for 1.1 bar fission chamber, which is close to the $150 \mathrm{~V}$ that Photonis [28] recommends as operation voltage for its CFUR43, considering a security margin. MCFD simulations show that for high neutron fluxes the saturation pla teau gets narrowed and the polarization voltage at saturation increases dangerously. Thus in order to measure high fluxes spe cial considerations for structural materials and filling gases should be taken. Chamber sensitivity is very close to the information available at [13]. MCFCD model accurately reproduces the most important parameters: fission rate, fission product yields, pairs production, variation of current signal, sensitivity and voltage of saturation. It also shows a correct sensibility to fluctuation in gas pressure and/or neutron fluxes. Small percentages of deviation from experimental data show that in some cases, MCFCD figures seem to be closer to measured data than other models.

In the new release, MCFCD will implement two new func tionalities, a) the effect of electromagnetic fields in the output signal of fission chambers, such as those present in space charge effect and magnetic confinement fields, b) directional neutron fluxes.

Table 4

Comparison of measured, calculated and simulated sensitivities for 1.1 and 4.0 bar subminiature fission chambers.

\begin{tabular}{|c|c|c|c|c|c|c|c|}
\hline$\left(\mathrm{A} / \mathrm{n} \mathrm{cm}^{2} \mathrm{~s}^{1}\right)$ & 1 bar & $1.1 \mathrm{bar}$ & Diff. (\%) & 2 bar & 3 bar & 4 bar & Diff. (\%) \\
\hline$S_{\text {measured }}[13]$ & & $1.1 \cdot 10^{17}$ & & & & $3.6 \cdot 10^{17}$ & \\
\hline$S_{\text {calculated }}[13]$ & & $0.7 \cdot 10^{17}$ & 36.4 & & & $2.8 \cdot 10 \quad 17$ & 22.2 \\
\hline$S_{M C F C D}$ & $9.8 \cdot 10^{18}$ & $0.95 \cdot 10^{17}$ & 13.6 & $1.9 \cdot 10^{17}$ & $2.9 \cdot 10^{17}$ & $3.8 \cdot 10^{17}$ & 5.6 \\
\hline
\end{tabular}




\section{Acknowledgments}

The authors would like to thank Dr. Philippe Filliatre from the CEA/Cadarache and Prof. Oscar Cabellos from the Nuclear Engi neering Department (Polytechnic University of Madrid) for their guidance and valuable recommendations concerning the design of fission chambers.

\section{References}

[1] R. Aymar, et al., Plasma Phys. Control. Fusion 44 (2002) 519.

[2] O.G. Egorov, V.V. Frunze, Yu. A. Kashchuk, At. Energy 106 (1) (2009) 50-59.

[3] G.F. Knoll, Radiation Detection and Measurement, third edition, John Wiley \& Sons, Inc (1999), p. 104-107.

[4] P. Filliatre, C. Jammes, B. Geslot, R. Veenhof, Nucl. Instrum. Methods Phys. Res, A 678 (2012) 139-147.

[5] D. Rapisarda, Á. García, Ó. Cabellos, F. Mota, J.M. Gómez-Ros, A. Ibarra, N. Casal, V. Queral, J. Sanz, Fusion Eng. Des. 84 (7-11) (2009) 1570-1574.

[6] D. Rapisarda, L. Vermeeren, Á. García, Ó. Cabellos, J.M. García, A. Ibarra, J. M. Gómez-Ros, F. Mota, N. Casal, V. Queral, Fusion Eng. Des. 86 (6-8) (2011) $1232-1235$.

[7] P. Filliatre, L. Vermeeren, C. Jammes, B. Geslot, D. Fourmentel, Nucl. Instrum. Methods Phys. Res. A 648 (2011) 228-237.

[8] The KornShell Command and Programming Language. 〈http://www.kornshell. $\mathrm{com} /\rangle$ (accessed 02.10.13).

[9] JANIS. 〈http://www.oecd-nea.org/janis/〉 (accessed 03.09.14).

[10] G.G. Raju, GASEOUS ELECTRONICS, Tables, Atoms, and Molecules, 24, CRC Press, Section I, Argon.

[11] The Stopping and Range of Ions in Matter. 〈http://www.srim.org (accessed 01.01.14).

[12] P. Filliatre, C. Jammes, B. Geslot, Nucl. Instrum. Methods Phys. Res. A 618 (2010) 294-297.

[13] O. Poujade, A. Lebrun, Nucl. Instrum. Methods Phys. Res. A 433 (1999) 673-682.
[14] Ö. Şahin, İ. Tapan, E.N. Özmutlu, R. Veenhof, J. Instrum. 5 (2010).

[15] Gnuplot home page, 〈http://gnuplot.info (accessed 03.09.14).

[16] O. Cabellos, P. Fernández, D. Rapisarda, N. García-Herranz, Nucl. Instrum. Methods Phys. Res. A 618 (2010) 248-259.

[17] G.G. Roussas, Introduction to Probability, second edition, Academic Press (2014), p. 31.

[18] W.M. Stacey, Nuclear Reactor Physics, second edition, WILEY-VCH Verlag GmbH \& Co.KGaA, Weinheim, 2007,, Pages [58, 60, 403].

[19] Sampling Distance to Next Collision. 〈http://mit-crpg.github.io/openmc/meth ods/physics.html $>$ (accessed 08.08.14).

[20] J. Devore. Probability and Statistics for Engineering and the Sciences, California Polytechnic State University, San Luis Obispo, Brooks/Cole, Cengage Learning, 2012, 2009, p. 138-142.

[21] W.M. Stacey, Nuclear Reactor Physics, second edition, WILEY-VCH Verlag GmbH \& Co.KGaA, Weinheim, 2007,, Pages [58, 60, 403].

[22] V.E. Viola, K. Kwiatkowski, M. Walker, Phys. Rev. C31 (1985) 1550.

[23] S. Chabod, G. Fioni, A. Letourneau, F. Marie, Nucl. Instrum. Methods Phys. Res. A 566 (2006) 633-653.

[24] S.N. Ahmed, Physics and Engineering of Radiation Detection, First edition, Academic Press an imprint of Elsevier (2007), p. 179.

[25] W. Blum, W. Riegler, L. Rolandi, Particle Detection with Drift Chambers, Springer-Verlag Berlin Heidelberg (2008), p. 157-165.

[26] F.H. Attix, Introduction to Radiological Physics and Radiation Dosimetry WILEY-VCH Verlag GmbH \& Co. KGaA (2004), p. 334, Chapter 12.

[27] R.L. Witkoverf, D. Gassner. Design of and Improved Ion Chamber for the SNS 〈http://www.bnl.gov/isd/documents/24068.pdf (accessed 03.09.14)

[28] CFUR43 Product specification. 〈http://www.photonis.com/attachment.php?id attachment 57> (accessed 02.10.14).

[29] Thermal flux spectrum. 〈http://nuclearpowertraining.tpub.com/h1019v1/img h1019v1_138_1.jpg (accessed 08.08.14).

[30] ICRU report 31, Average Energy Required to Produce an Ion Pair, 1979.

[31] H.W. Koch and R.W. Johnston. Multichannel Pulse Height Analyzers: Proceedings of an Informal Conference, National Academy of Sciences - National Research Council (U.S.). Committee on Nuclear Science, Gatlinburg, Tennessee, September 26-28, 1956, p. 13.

[32] T. Nicholas., Measurement and Detection of Radiation, 2nd ed., Taylor \& Francis (1995), p. 183-185. 